\title{
Über Isobutylnaphtalin.
}

\author{
(Vorläufige Mittheilung.)
}

\section{Von Dr. Rudolf Wegscheider.}

(Aus dem Universitïtslaboratorium des Prof. v. Barth in Wien.)

(Vorgelegt in der Sitzung am 23. Mai 1884.)

Die Frage, ob im Phenanthren jene zwei Kohlenstoffatome, welche bei der Überfiihrung dieses Kohlenwasserstoffs in Diphenyl abgespalten werden, mit vier andern zusammen einen Benzolkern bilden, respective, ob im Phenanthren sich drei Gruppen von je sechs Kohlenstoffatomen nachweisen lassen, welche in derselben Beziehung zu einander stehen, wie die sechs Kohlenstoffatome des Benzols, ist bisher experimentell nicht entschieden worden. Einen Weg zur Lösung dieser Frage habe ich bereits vor längerer Zeit angedeutet; ${ }^{1}$ gelänge es, Naphtalin in Phenanthren überzuführen, so wäre damit die bejahende Antwort gegeben. Seither haben Skraup und Vortmann ${ }^{2}$ aus $m$ - und $p$-Phenylendiamin zwei Körper dargestellt (Phenanthrolin und Pseudophenanthrolin), welche bei der Oxydation sich ganz analog dem Phenanthren verhalten, indem sie dabei in Dipyridyldicarbonsäuren übergehen, welche ihrerseits wieder durch Kohlensäureabspaltung in Dipyridyle tiberfuhrhar sind. Aus der Entstehung der Phenanthroline folgt zweifellos, dass jene zwei Kohlenstoffatome, welche bei der Überführung in Dipyridyle abgespalten werden, Glieder eines Benzolkernes sind. Ferner haben Skraup und Cobenzl ${ }^{3}$ aus den beiden Naphtylaminen die Naphtochinoline dargestellt, welche, ebenfalls in vollständiger Analogie mit der Bildung von Diphensäure und Diphenyl aus Phenanthren, in Phenylpyridindicarbon-

1 Mon. f. Chemie, I., 917.

2 Mon. f. Chemie, III, $570 \mathrm{fi}$, IV., $569 \mathrm{ff}$.

3 Mon. f. Chemie, IV., $436 \mathrm{ff}$. 
säuren und Phenylpyridine ïberfilhrbar sind. Diese Analogien machen es wahrscheinlich, dass die oben bezüglich des Phenanthrens formulirte Frage bejahend zu beantworten und das Phenanthren sowohl als Derivat des Diphenyls, als auch des Naphtalins aufzufassen ist. Nichtsdestoweniger schien es mir bei dem Interesse, welches der Gegenstand für die Theorie der Kohlenwasserstoffe mit condensirten Benzolkernen hat, nicht iiberflüssig, die Erbringung eines directen Beweises für die Auffassung des Phenanthrens als Naphtalinderivat zu versuchen.

Am geeignetsten erscheinen hiezu die Isobutylnaphtaline, da deren Überfïhrbarkeit in Phenanthren nach der durch Wreden und Znatovich ${ }^{1}$ nachgewiesenen Bildung von Naphtalin aus Isobutylbenzol wahrscheinlich und mindestens eines der: beiden Isomeren leicht zu erhalten ist. Ich habe daher vor einiger Zeit die Darstellung dieser Kohlenwasserstoffe begonnen. Nun hat soeben Herr Léon $\mathrm{R}_{0} \mathrm{ux}^{2}$ eine Mittheilung veröffentlicht, derzufolge er mit dem Studium der Einwirkung der Chloride, Bromide und Jodide der Alkoholradikale auf Naphtalin in Gegenwart von Aluminiumchlorid beschäftigt ist. Obwolll Herr Roux die Isobutylnaphtaline bisher nicht in den Kreis seiner Untersuchung einbezogen hat, will ich doch nicht unterlassen, mir dureh die Beschreibung meiner bisher gemachten Beobachtungen das Recht des Weiterarbeitens zu sichern, umsomehr, da ich durch eine unaufsehiebbare grössere Arbeit fuir einige Zeit verhindert bin, auf die Fortführung der erwähnten Versuche mein Augenmerk zu richten.

Die Anwendung der schönen Methode von Friedel und Crafts ermöglicht es, aus Naphtalin und Isobutylchlorid ohne Schwierigkeit ein Isobutylnaphtalin zu erhalten. Da diese beiden Körper behufs Erzielung einer guten Ausbeute möglichst innig gemischt sein müssen, das feste Naphtalin sich aber selbst in kochendem Isobutylehlorid schwer löst, so habe ich zterst zwei Theile Naphtalin zum Schmelzen erhitzt und dann einen Theil Isobutylchlorid hinzugefügt. Man erhält so eine iibersättigte

1 Ber. Deutsch. Ch. Ges., IX., 1606.

2 Sur la préparation d'une propyle et d'une amylnaphtaline, Bull. de la soc. chim. de Paris, tome XLI, Nro. 8 (20 avril 1884). p. 379. 
Lösung, welche erst bei starkem Sinken der Temperatur das Naphtalin in Nadeln auskrystallisiren lässt. In diese Lösung wurde in kleinen Portionen Aluminiumchlorid eingetragen und dabei nur soweit erwärmt, als nöthig war, um das Auskrystallisiren zu verhindern. Es entwichen Ströme von Chlorwasserstoff, ausserdem trat in dem mit dem Riickflusskühler verbundenen und mit Brom und Wasser gefüllten Péligot'schen Apparat eine kleine Menge eines Öles auf. Als weiterer Zusatz von Chloraluminium bei der eingehaltenen Temperatur keine Gasentwicklung mehr bewirkte, wurde die Operation unterbrochen; dieser Punkt trat ein, als das Gewicht des eingetragenen Chloraluminiums etwa ein Zwölftel von dem des angewendeten Naphtalins betrug. Nun wurde der dunkel gefärbte Kolbeninhalt in eine tubulirte Retorte ibergefuihrt und der Destillation mit gespannten Wasserdämpfen unterworfen. Hiebei ging zuerst unverändertes Naphtalin ïber, dann Isobutylnaphtalin als farbloses Öl, endlich in geringer Menge weisse Krystalle. Diese wurden aus starkem Weingeist umkrystallisirt und dadurch in zwei Substanzen zerlegt, von denen die eine feine Nadeln (Schmelzpunkt ungefähr $80^{\circ}$ ), die andere Blättchen oder wohlausgebildete Prismen (Schmelzpunkt 146-147 ${ }^{\circ}$ ) bildete; die letztere entsteht nur in ganz geringer Menge. Vermuthlich ist die erstere identisch mit dem $\alpha$ - $\beta$-Dinaphtyl, die andere mit dem $\alpha$ - $\alpha$-Dinaphtyl. Der nach dem Destilliren mit Wasserdampf in der Retorte bleibende Riickstand wurde unter vermindertem Druck destillirt und das Destillat aus Alkohol umkrystallisirt. So erhielt ich bei $188^{\circ}$ schmelzende gelbe Blättchen oder Nadeln; sie lösen sich beim Erhitzen in concentrirter Schwefelsäure mit blaugrüner Farbe, die beim Stehen misfärbig wird; nach Zusatz von Salpetersäure geht sie in roth iiber. Es liegt also Isodinaphtyl vor. Dementsprechend wurde die Dampfdichte zu $9 \cdot 02$ gefunden (berechnet für $\mathrm{C}_{20} \mathrm{H}_{14} 8 \cdot 78$ ). Die Bildung von Isodinaphtyl hat Roux ${ }^{l}$ auch bei der Darstellung des Propyl- und Amylnaphtalins beobachtet.

Das erhaltene, noch nicht absolut reine Isobutylnaphtalin ist ein farbloses, mit Wasserdampf nur schwer flüchtiges Öl, welches

1 In der eingangs citirten Abhandlung. 
bei gewöhnlichem Druck ungefähr bei $280^{\circ}$ siedet und sich leicht in Äther löst. Seine Pikrinsäureverbindung bildet bei $96^{\circ}$ schmelzende, in Alkohol leicht lösliche, gelbe, kugelförmige Aggregate von feinen Nadeln. Die Gegenwart einer isomeren Verbindung hat bisher nicht nachgewiesen werden können.

Auch wenn man Naphtalin mit Aluminiumchlorid am Wasserbade erwärmt, bis der Kolbeninhalt völlig geschmolzen ist, and dann portionenweise Isobutylchlorid einträgt, erhält man Isobutylnaphtalin, jedoch in schlechterer Ausbente. $\mathrm{R}$ oux ${ }^{\prime}$ hat bei einem analogen mit Amylchlorid, jedoch bei höherer Temperatur $\left(120^{\circ}\right)$ angestellten Versuch gar kein Amylnaphtalin, dagegen reichlich Isodinaphtyl und Pentan erhalten; er betont, dass die Bildung des Dinaphtyls aus Naphtalin mittelst Chloraluminium bei Gegenwart eines Alkylchlorids viel reichlicher eintritt, als ohne dieselbe. Auch ich habe mich davon ïberzengt, indem ich Aluminiumchlorid auf Naphtalin allein einwirken liess; dabei entstanden nur sehr geringe Mengen von Dinaphtyl. Es liegt die Vermuthung nahe, dass die gesteigerte Bildung von Dinaplityl bei Gegenwart des Chlorids eines Alkoholradikales darauf berult, dass zuerst der entsprechende Kohlenwasserstoff (Butyl-, respective Amylnaphtalin) entsteht, dieser aber durch überschüssiges Chloraluminium wieder zersetzt wird, etwa nach der Gleichung

$$
\mathrm{C}_{10} \mathrm{H}_{7}\left(\mathrm{C}_{4} \mathrm{H}_{9}\right)+\mathrm{C}_{10} \mathrm{H}_{8}=\mathrm{C}_{20} \mathrm{H}_{14}+\mathrm{C}_{4} \mathrm{H}_{10} \text {. }
$$

Dass Chloraluminium unter Umständen Seitenketten abspaltet, ist von Friedel und $\mathrm{Crafts}$ gezeigt worden; z. B. wird Hexamethylbenzol in Durol tibergeführt. ${ }^{2}$ Ist diese Auffassung: des Vorganges zutreffend, so muss bei der Darstellung der Homologen des Naphtalins die Gegenwart eines grösseren Überschusses von Aluminiumchlorid vermieden werden. Thatsächlich erhält man, wie bereits erwähnt, schlechte Ausbeuten, wenn man das Alkylchlorid zu dem Gemisch von Naphtalin und Aluminiumchlorid setzt, letzteres also in der Reactionsmasse stets im Überschuss vorhanden ist, und zwar um so schlechtere, bei je höherer Temperatur man die Reaction einleitet. In demselben Sinne

1 In der eingangs citirten Abhandlung.

2 Ber. Deutsch. Ch. Ger., XV, 1451. 
lässt sich folgende Beobachtung denten. Als ich bei einer Darstellung des Isobutylnaphtalins den Zusatz von Aluminiumchlorid iiber den bei der Beschreibung der Methode angegebenen Zeitpunkt hinaus unter Steigerung der Temperatur fortsetzte, begann eine beträchtliche Menge eines brennbaren, durch Brom nicht absorbirten Gases zu entweichen, wahrscheinlich Butan. Die Ausbeute an Isobutylnaphtalin war bei dieser Operation nicht befriedigend.

Ausser der Friedel-Craft'schen Methode haba ich zur Darstellung des Isobutylnaphtalins auch jene Methode anzuwenden versucht, welche Heinrich Golds chmidt zur Darstellung von Homologen des Benzols beniitzt hat ${ }^{1}$ (Einwirkung eines Alkohols auf Benzol in Gegenwart von Zinkchlorid), da der Isobutylalkohol ein vortheilhafteres Ansgangsmaterial ist als das Chlorid. Die Fractionen des Reactionsproductes zwischen Naphtalin, Isobutylalkohol und Chlorzink jedoch, welche ihrem Siedepunkt nach das Isobutylnaphthalin hätteı enthalten miissen, gaben keine Pikrinsäurererbindung und bestanden daher wohl grösstentheils aus Condensationsproducten des Alkohols.

1 Ber. Dentsch. Ch. Ges., XV., 1066, 1425. 